\title{
Effets des antioxydants (éthoxiquine et BHT) sur la stabilité de la canthaxanthine au cours de la granulation; conséquences sur la pigmentation de la truite arc-en-ciel
}

\author{
G. CHOUBERT \\ avec la collaboration technique de Laurence BarraU, R. Cescosse, \\ Y. HONTANG et R. LANNEBERE \\ I.N.R.A., Laboratoire de Nutrition et d'Elevage des poissons \\ Contre de Recherches hydrobiologiques \\ Saint-Pée-sur-Nivelle, F 64310 Ascuin
}

\section{Résumé}

On a étudié l'effet de deux antioxydants, l'éthoxyquine et le Butyl-Hydroxy-Toluène (BHT), sur la stabilité de la canthaxanthine lors du pressage de l'aliment et sur la pigmentation de la truite arc-en-ciel. A cette fin, un aliment pour truite a été supplémenté en antioxydant aux doses de $0,50,100,150 \mu \mathrm{g} / \mathrm{g}$ de substance active.

Le processus de granulation entraîne une destruction de la canthaxanthine de l'ordre de 10 p. 100 quel que soit l'antioxydant utilisé. On note toutefois que les plus fortes supplémentations en antioxydant $(150 \mu \mathrm{g} / \mathrm{g})$ donnent les meilleurs résultats.

Dans nos conditions expérimentales nous n'avons mis en évidence aucune action significative des antioxydants sur la pigmentation de la truite arc-en-ciel.

Mots clés : antioxydant, canthaxanthine, pigmentation, truite.

\section{Introduction}

Chez la truite arc-en-ciel, la rétention musculaire des pigments caroténoïdes est basse, de l'ordre de 1 à 2 p. 100 (Schiedt \& Leuenberger, 1981; Choubert \& LuQuet, 1982) et ne représente que le dixième de ce qui est observé chez le poulet (Chemillier, 1977).

Compte tenu de la lente mobilisation de la canthaxanthine chez le poisson (Choubert, 1984), l'explication de ce phénomène passe par l'analyse des facteurs pouvant intervenir avant la fixation de ce pigment dans les tissus : disponibilité, digestibilité, métabolisation.

La canthaxanthine étant un système polyénique fortement conjugué, sa destruction peut, dès lors, avoir lieu au cours des processus de fabrication de l'aliment. En effet, la granulation de l'aliment exerce un rôle de fragilisation de l'enrobage par 
laminage des particules contre la filière (VALDEBOuze \& LEVY, 1972). La détérioration ainsi subie, hâterait la destruction du pigment par suite de la considérable surface exposée à l'air. Ces pertes au cours de la granulation peuvent atteindre 20 p. 100 (Choubert \& Luquet, 1979). Ce problème est d'autant plus important que les conditions et la durée de conservation des aliments peuvent apporter des modifications sensibles, difficilement prévisibles, étant donné la variété des formules alimentaires, les conditions de fabrication et de distribution des aliments (MAINGUY \& Rououes, 1965).

Choubert \& Luouet (1979) ont fait apparaître, en moyenne, une faible digestibilité de la canthaxanthine chez la truite arc-en-ciel, de l'ordre de 20 p. 100 . Ils montrent de plus, qu'il existe une forte variabilité entre les échantillons pour un même lot de fabrication de ce pigment. Or la digestibilité mesurée par ces auteurs est une digestibilité apparente, c'est-à-dire ne tenant pas compte de la labilité de la canthaxanthine. L'éventualité d'une dégradation de ce pigment peut cependant être retenue comme facteur d'une mauvaise digestibilité car on sait qu'une dégradation des caroténoïdes se produit au cours du transit digestif (Bоотн, 1956).

Les antioxydants ont, de plus, été utilisés pour accentuer la pigmentation du poulet de chair ou celle du jaune de l'œuf. Toutefois l'effet de cette supplémentation est très variable. Tantôt la présence d'antioxydant défavorise la pigmentation du poulet de chair, tantôt elle la favorise. Dans ce cas, les teneurs en pigments caroténoïdes fixés peuvent être augmentées de 5 à 40 p. 100 (MARusich \& BaUernfiend, 1981). Le mécanisme d'action des antioxydants n'est pas encore élucidé.

Aucun travail n'ayant été rapporté dans ce domaine chez le poisson, nous avons donc entrepris l'étude, objet de la présente publication, de l'effet de deux antioxydants, l'éthoxyquine et le B.H.T. sur la stabilité de la canthaxanthine au cours de la granulation ainsi que sur la pigmentation de la truite arc-en-ciel.

\section{Matériel et méthodes}

\section{A. Antioxydants}

Les deux antioxydants utilisés ont été choisis parmi ceux d'un emploi courant en alimentation animale. La forme chimique et la présentation sont celles de préparations commerciales ttsuelles, à savoir :

66 p. 100 ;

- l'éthoxyquine (6-éthoxy-2, 2, 4-triméthyl-1, 2-dihydroquinoléine) pureté

- le B.H.T. (di-tertio-butyl-hydroxy-toluène) pureté 99 p. 100.

Chacune de ces préparations est introduite dans un aliment expérimental (1) de façon à réaliser des concentrations de $0,50,100$ et $150 \mathrm{mg}$ de substance active par kilogramme.

(1) Composition de l'aliment expérimental en g/p. $100:$ Farine de poisson du Danemark : 80 ; Blé : 16,5»; Huile de poisson : 1 ; Complément minéral (LuQuet, 1971) : 0,5; Complément vitaminique (EIFAC, 1971) dont acétate de DL- $\alpha$-tocophérol 0,5 p. $100: 2$. 


\section{B. Aliments}

Les régimes alimentaires sont supplémentés en canthaxanthine de façon à réaliser une concentration d'environ $200 \mathrm{mg} / \mathrm{kg}$ de produit pur. Les aliments sont granulés par une presse de laboratoire C.P.M. avec adjonction d'eau ( 2 p. 100 du mélange). Les aliments sont conservés dans des sacs en papier, stockés sous abris, à température ambiante et utilisés dans les 40 jours qui ont suivi leur fabrication.

\section{Poissons}

L'expérience d'une durée de 27 jours s'est déroulée à la pisciculture expérimentale de Donzacq (Landes). 8 lots de 100 truites arc-en-ciel (Salmo gairdneri Rich.) d'un poids initial de $120 \mathrm{~g}$ sont constitués au hasard et placés dans des bassins rectangulaires de $2 \mathrm{~m}^{2}$ de surface et $0,50 \mathrm{~m}$ de hauteur d'eau. Ces bassins sont alimentés en eau de source $\left(17^{\circ} \mathrm{C}\right)$ à un débit de $360 \mathrm{l} / \mathrm{min}$. Les truites sont nourries à volonté par repas, deux fois par jour.

En fin d'expérience, un échantillon de 10 truites est retenu pour analysc. Celles-ci sont tuées par rupture des vertèbres cervicales puis pesées. La musculature épaxiale provenant des différents poissons est prélevée, congelée, regroupée et conservée dans l'obscurité et au froid $\left(-18^{\circ} \mathrm{C}\right)$ jusqu'au broyage qui précède immédiatement les analyses.

\section{Déterminations analytiques}

\section{Lipides totaux}

Le dosage des lipides totaux est effectué selon la méthode décrite par Folch, LeEs \& Sloane-Stanley (1957) sur des prises d'essai de $10 \mathrm{~g}$ de produit frais.

\section{Canthaxanthine des aliments}

La canthaxanthine contenue dans les aliments est extraite sclon le protocole établi par Osadca, Araujo \& DE RitTer (1972) sur une prise d'essai de $15 \mathrm{~g}$ de produit frais. Le dosage s'effectue comme suit : $20 \mathrm{ml}$ de la solution d'extraction sont prélevés; le mélange de solvants évaporé est remplacé par $10 \mathrm{ml}$ d'acétone que l'on congèle $\left(-80^{\circ} \mathrm{C}\right)$ puis filtré sur verre fritté (porosité $5-15 \mu \mathrm{m}$ ) de façon successive (Müller-Mulot, Rohrer \& Medweth, 1976). La densité optique de l'extrait ainsi obtenu est lue à $472 \mathrm{~nm}$, longueur d'onde du maximum d'absorption de la canthaxanthine dans ce solvant $\left.\mathrm{E}_{1 \% \mathrm{~m}}^{1 \%}=2100\right)$.

\section{Canthaxanthine du muscle du poisson}

Cette détermination s'effectue sur $2 \mathrm{~g}$ de muscle frais. Le muscle est finement broyé dans $48,5 \mathrm{ml}$ d'acétone pendant 3 minutes. Cette concentration d'acétone (97 p. $100 \mathrm{v} / \mathrm{v}$, en tenant compte de l'eau contenue dans les tissus) a été retenue, après essais antérieurs, pour son pouvoir d'extraction optimum de la canthaxanthine. L'extrait ainsi obtenu est alors filtré sur papier whatman 1 P.S. Le volume de la solution filtrée est exactement mesuré et la mesure spectrophotométrique s'effectue dans les mêmes conditions que pour la canthaxanthine dans l'aliment. 


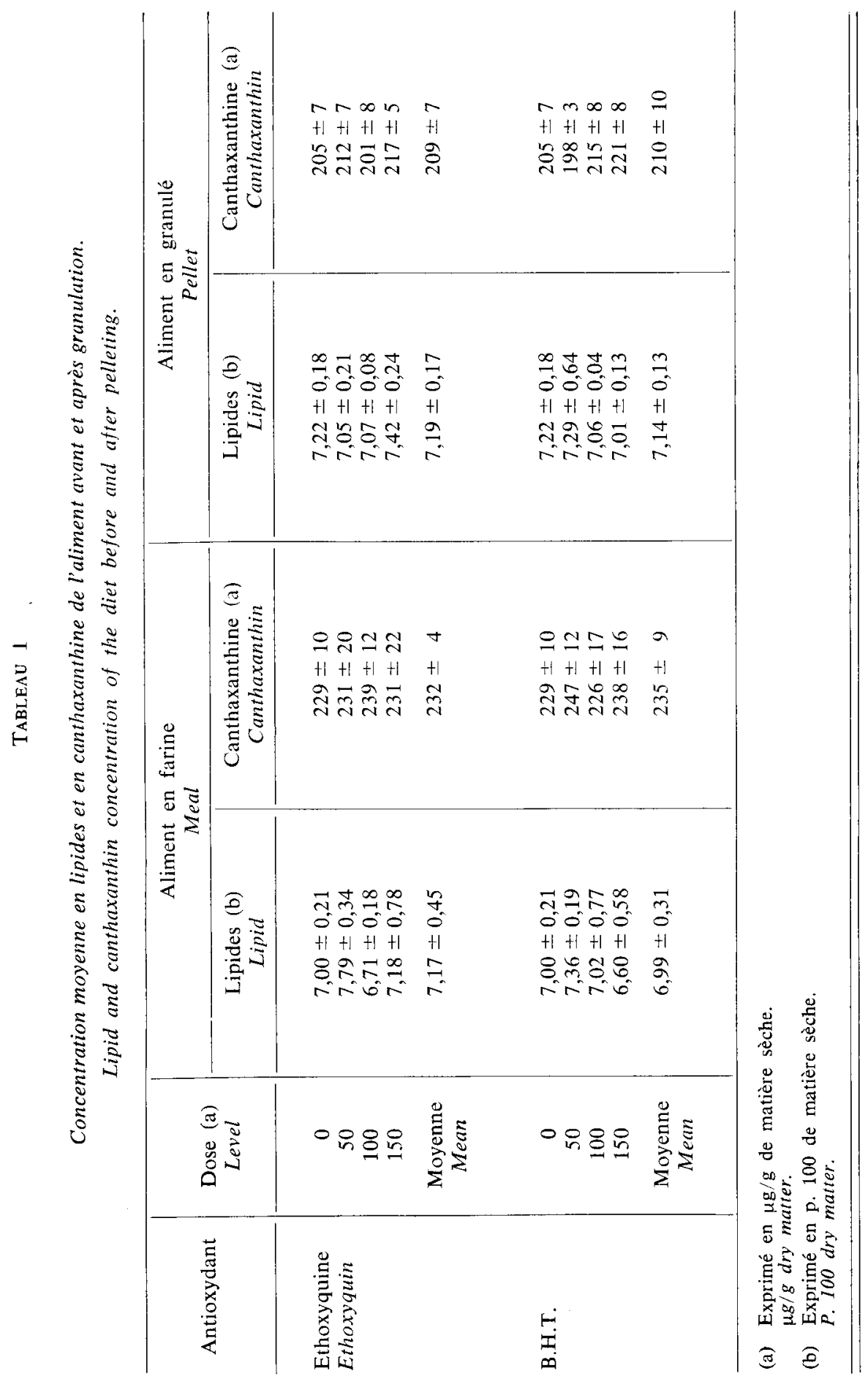


4. Tests statistiques

Nous avons utilisé la méthode classique d'analyse de la variance décrite par SNEDECOR \& COCHRAN (1967).

\section{Résultats}

A. Stabilité de la canthaxanthine (Tabl. 1 et 2)

On vérific tout d'abord que les teneurs en lipides de l'aliment tant en farine qu'en granulé sont homogènes (de l'ordre de 7 p. 100 M.S.) quels que soient l'antioxydant et la dose utilisés.

\section{TABLEAU 2}

Effets de l'antioxydant sur la stabilité de la canthaxanthine au cours de la granulation : analyse de variance.

Effects of antioxydant on the stability of the canthaxanthin during the pelleting process: analysis of variance.

\begin{tabular}{|c|c|c|c|c|}
\hline $\begin{array}{l}\text { Source de variation } \\
\text { Source of variation }\end{array}$ & $\begin{array}{l}\text { Degré } \\
\text { de liberté } \\
\text { Degree } \\
\text { of freedom }\end{array}$ & $\begin{array}{l}\text { Carré moyen } \\
\text { Mean square }\end{array}$ & $F$ & $\begin{array}{l}\text { Signification } \\
\text { Significance }\end{array}$ \\
\hline Antioxydant $\ldots \ldots \ldots$ & 1 & 14.22 & $<1$ & $\mathbf{N S}(*)$ \\
\hline $\begin{array}{l}\text { Dose } \\
\text { Level }\end{array}$ & 3 & 137,51 & 1.05 & NS \\
\hline $\begin{array}{l}\text { Agglomération .......... } \\
\text { Pelleting process }\end{array}$ & 1 & 4917,10 & 37.8 & HS $(* *)$ \\
\hline Interactions & & & & \\
\hline $\begin{array}{l}\text { Dose/antioxydant } \\
\text { Level/antioxydant }\end{array}$ & 3 & 11,29 & $<1$ & NS \\
\hline $\begin{array}{l}\text { Agglomération/antioxydant } \ldots . . \\
\text { Pelleting process/antioxydant }\end{array}$ & 1 & 5,07 & $<1$ & NS \\
\hline $\begin{array}{l}\text { Dose/agglomération .......... } \\
\text { Level/pelleting process }\end{array}$ & 3 & 113,16 & $<1$ & NS \\
\hline $\begin{array}{l}\text { Antioxydant/dose/agglomération } \\
\text { Antioxydant/level/ } \\
\text { pelleting process }\end{array}$ & 3 & 277,63 & 2.13 & NS \\
\hline $\begin{array}{l}\text { Résiduelle } \\
\text { Residual }\end{array}$ & 16 & 130,07 & & \\
\hline
\end{tabular}

(*) Non significatif - Not significant.

(**) Hautement significatif - Highly significant $(\mathrm{P}<0,001)$. 
Si les valeurs moyennes de concentration en canthaxanthine sont relativement homogènes pour l'aliment en farine (environ $230 \mu \mathrm{g} / \mathrm{g}$ M.S.) quels que soient l'antioxydant ef la dose employés, celles notées dans les granulés sont plus variables : de 201 à $217 \mu \mathrm{g} / \mathrm{g}$ M.S. pour l'aliment contenant de l'éthoxyquine et 198 à $221 \mu \mathrm{g} / \mathrm{g}$ M.S. pour celui renfermant du BHT. On remarque, de plus, que selon la dose d'antioxydant utilisée, la concentration en canthaxanthine dans les granulés est différente. Cette différence atteint 5,8 p. 100 pour l'éthoxyquine et 7,8 p. 100 pour le BHT entre les doses de 0 à 150 . Il est à noter, de plus, que les écarts sont plus importants dans les résultats de concentration en canthaxanthine de la farine que dans ceux des granulés.

En procédant à l'analyse de variance entre les résultats obtenus pour les concentrations en canthaxanthine de l'aliment en farine et en granulé, on observe un effet positif significatif au seuil de 1 p. 100 de la supplémentation en antioxydant sur la stabilité de la canthaxanthine lors de la granulation de l'aliment.

\section{B. Pigmentation des truites (Tabl. 3 et 4)}

On note que le caractère cssentiel des résultats de concentration en canthaxanthine obtenus est un coefficient de variation toujours très élevé.

On constate, de plus, que les supplémentations les plus fortes ne tendent pas à donner les résultats les meilleurs. Ainsi, dans le cas de l'utilisation de l'éthoxyquine, les concentrations en canthaxanthine du muscle sont de $9,2 \mu \mathrm{g} / \mathrm{g}$ de M.S. pour la dose 150 et $10,7 \mu \mathrm{g} / \mathrm{g}$ M.S. pour la dose 0 . Cependant pour le BHT, la tendance est un peu différente $(8,6 \mu \mathrm{g} / \mathrm{g}$ M.S. pour la dose 0 et 9,7 $\mu \mathrm{g} / \mathrm{g}$ M.S. pour la dose 150).

\section{TABLEAU 3}

Concentration en lipides et en canthaxanthine de la musculature épaxiale du poisson.

Lipid and canthaxanthin concentration of muscle.

\begin{tabular}{l|c|c|c}
\hline \hline \multicolumn{1}{c|}{ Antioxydant } & $\begin{array}{c}\text { Dose (a) } \\
\text { Level }\end{array}$ & $\begin{array}{c}\text { Lipides (b) } \\
\text { Lipid }\end{array}$ & $\begin{array}{c}\text { Canthaxanthine (a) } \\
\text { Canthaxanthin }\end{array}$ \\
\cline { 2 - 4 } Ethoxyquine & 0 & $16,58 \pm 0,84$ & $10,7 \pm 0,6$ \\
Ethoxyquin & 50 & $15,17 \pm 1,03$ & $11,2 \pm 0,7$ \\
& 100 & $17,45 \pm 0,02$ & $9,4 \pm 0,6$ \\
& 150 & $16,48 \pm 0,47$ & $9,2 \pm 0,3$ \\
& Moyenne & $16,42 \pm 0,94$ & $10,1 \pm 0,91$ \\
Bean & & & \\
& 0 & $15,51 \pm 0,71$ & $8,6 \pm 0,2$ \\
& 50 & $16,70 \pm 1,18$ & $6,4 \pm 0,7$ \\
& 100 & $18,82 \pm 0,16$ & $5,4 \pm 0,7$ \\
& 150 & $17,45 \pm 0,31$ & $9,7 \pm 0,2$ \\
& Moyenne & $17,32 \pm 1,38$ & $7,5 \pm 1,89$ \\
\hline
\end{tabular}

(a) Exprimé en $\mu \mathrm{g} / \mathrm{g}$ de matière sèche $-\mu g / g$ dry matter.

(b) Exprimé en p. 100 de matière sèche $-P .100$ dry matter. 
TABLEAU 4

Effet de l'antioxydant et de la dose sur la pigmentation du muscle de truite: analyse de variance.

Effect of antioxydant on the pigmentation of the rainhow trout muscle : analysis of variance.

\begin{tabular}{|c|c|c|c|c|}
\hline \multirow[b]{2}{*}{ Dose - Level ..... } & \multirow{2}{*}{$\begin{array}{l}\text { Somme des carrés } \\
\text { Sum of squares } \\
4,43\end{array}$} & \multirow{2}{*}{$\begin{array}{c}\text { Degré de liberté } \\
\text { Degrec of freedom } \\
\frac{3}{3}\end{array}$} & \multicolumn{2}{|c|}{$\mathrm{F}$} \\
\hline & & & 0,41 & $N S(*)$ \\
\hline Antioxydant $\ldots \ldots$ & 17,94 & 1 & 5,02 & NS \\
\hline Erreur - Error .. & 10,72 & 3 & 一 & - \\
\hline Total . . & 33,09 & 7 & & \\
\hline
\end{tabular}

(*) NS = non significatif - Not significant.

En procédant à l'analyse de variance des résultats obtenus pour la canthaxanthine du muscle des poissons, le calcul statistique ne fait pas apparaître de signification particulière entre l'antioxydant utilisé ou la dose employée.

\section{Discussion}

La canthaxanthine $\left(\beta, \beta\right.$-carotène-4, $4^{\prime}$-dione) est un constituant naturel de la nourriture des salmonidés qui provoque une pigmentation rouge de leur chair et de leur robe (Thommen \& Gloor, 1965 ; Auger, 1973). Ce pignent synthétisé industriellement (Isler, Ofner \& Siemers, 1958) est depuis couramment utilisé en pisciculture intensive.

Compte tenu de sa fragilité, la canthaxanthine nécessite une protection vis-à-vis des phénomènes d'oxydation. Celle-ci est effectuée dans les concentrats soit par enrobage du pigment par de la gélatine soit par microdispersion dans un support glucidique. Ce mode de protection est efficace et limite les pertes en canthaxanthine à 0,5 p. 100 par mois (Bunnel \& Borenstein, 1967 ; Choubert \& Luquet, 1979).

La granulation entraîne une diminution de la teneur en canthaxanthine dans l'aliment. Cette diminution atteint dans nos conditions expérimentales 10 p. 100, ce qui ne représente que la moitié de ce qui a été rapporté par ChouberT \& LuoueT (1979). L'utilisation d'antioxydants tels que l'éthoxyquine ou le BHT peut donc s'avérer positive. Toutefois, la différence de formulation alimentaire ne doit pas être exclue. En effet, dans cette expérience, l'aliment contenait 80 p. 100 de farine de poisson et 16,5 p. 100 de blé broyé. Cette formulation simple a permis une granulation aisée sans contraintes particulières. Par contre l'aliment utilisé antérieurement (Choubert \& Luouet, 1979) était plus complexe (blé 33 p. 100, tourteau de soja 22 p. 100, farine de poisson 35 p. 100). Il se peut que le laminage des particules contre les parois de la filière ait été plus important. 
Dans nos résultats expérimentaux, on remarque que les écarts types des concentrations en canthaxanthine sont plus faibles pour l'aliment en granulé que pour l'aliment en farine. Cette observation traduit ainsi le fait que la granulation d'une part est un facteur de plus dans l'homogénéisation de l'aliment et d'autre part semble favoriser l'extraction ultérieure de la canthaxanthine, probablement par fragilisation de l'enrobage contre les parois de la filière (VALDEbouze \& LeVy, 1972). Cette observation, de plus, conforte la notion même du rôle des antioxydants vis-à-vis de la stabilité de la canthaxanthine dans les aliments : une action d'autant plus faible que lcs conditions de granulation sont douces, c'est-à-dire que la fragilisation de l'enrobage est, elle-même, plus faible.

Nous n'avons pu mettre en évidence, dans nos conditions expérimentales, aucune action des antioxydants sur la pigmentation de la truite arc-en-cicl. Les résultats enregistrés sont très hétérogènes et semblent relever beaucoup plus d'aléas de l'expérimentation que de quclconques effets des antioxydants. Cette variabilité des réponses n'est pas le seul fait des poissons et se retrouve également chez le poulet de chair (MARUSICH \& BAUERNFEIND, 1981). Elle peut s'expliquer par la multiplicité des paramètres souvent mal contrôlés qui agissent sur la pigmentation des animaux, paramètres relatifs aux animaux, aux aliments et aux conditions d'élevage. Selon MARUSICH \& BAUERNFEIND (1981) les antioxydants agiraient davantage par une protection de la canthaxanthine « in vitro » donc dans l'aliment plutôt que par une quelccnque action « in vivo", chez l'animal. Il en résulterait une augmentation de la quantité de pigment mise à la disposition de l'animal. La digestibilité de ce pigment deviendrait dès lors le facteur essentiel. C'est ce qu'il conviendra de vérifier.

Reçu en octobre 1984. Accepté en novembre 1984.

\section{Remerciements}

A la Société F. Hoffmann-La Roche et C" pour la fourniture de l'éthoxyquine et de la canthaxanthine et à la Société Française d'Organo-Synthèse pour la fourniture du B.H.T.

\section{Summary}

Effects of antioxydants (ethoxyquin and B.H.T.) on the stability of canthaxanthin during the pelleting process; consequences on the rainbow trout pigmentation

The effects of two antioxydants, ethoxyquin and B.H.T., on the stability of the canthaxanthin during the pelleting process and on the pigmentation of the rainbow trout were studied.

For that purpose, a diet for rainbow trout was supplemented with $0,50,100,150 \mu \mathrm{g} / \mathrm{g}$ of antioxydant (ethoxyquin or B.Н.Г.).

Pelleting process involves destruction of $\mathrm{Ca} 10 \mathrm{p} .100$ of the canthaxanthin, whatever the antioxydant used (tables 1 and 2). However, the protection of canthaxanthin was larger with the highest level of antioxydant $(150 \mu \mathrm{g} / \mathrm{g})$.

In our experimental conditions, antioxydants seemed to have no effect on canthaxanthin deposition in the muscle of rainbow trout (tables 3 and 4).

Key words : antioxydant, canthaxanthin, pigmentation, trout. 


\section{Références bibliographiques}

Augle G.A.H., 1973. La canthaxanthine, son influence sur la coloration de la chair des truites, thèse Doc. Vét., Paris-Créteil, 112 p.

Bоотн V.H., 1956. Disappearance of carotene from the alimentary tract of vitamin A deficient rats. Br. J. Nutr., 10, 241-250.

BunNel R.H., Borfanstian B., 1967. Canthaxanthin, a potential new food color. Food Technol., 21, 331-334.

Chemillier J., 1977. La pigmentation du poulet de chair. Doc Roche, 1393, 35 p.

Chonbert G., 1984. Effects of starvation and feeding on canthaxanthin depletion of muscle in rainbow trout (Salmo gairdneri Rich). Aquaculture (sous presse).

Choubert G., Luquer P., 1979. Influence de l'agglomération et du stockage des aliments composés sur leur tencur en canthaxanthine : conséquence sur la digestibilité et la fixation de ce pigment chez la truite arc-en-ciel. Ann. Zootech., 28, 145-157.

Choubert G., Luquet P., 1982. Fixation et rétention musculaire de la canthaxanthine par la truite arc-en-ciel. Ann. Zootech., 31, 1-10.

E.I.F.A.C., 1971. Salmon and trout feeds and feeding. E.I.F.A.C. Techn. Pap., 12, 29 p.

Folch J., Lees M., Sloanf-Stanley G.H., 1957. A simple method for the isolation and purification of total lipids from animal tissue. J. Biol. Chem., 226, 497-509.

IsLer O., OfNer A., Siemers G.F., 1958. Industrial syntheses of carotenoids for use as food colors. Food technol., 12, 520-526.

LUQueT P., 1971. Efficacité des protéines en relation avec leur taux d'incorporation dans l'alimentation de la truite arc-en-ciel. Ann. Hydrobiol., 2, 175-186.

Mainguy P., Rouques A., 1965. Le jaune de l'œuf : I. Etude générale de sa couleur. Bull. Soc. Scient, Hyg. Aliment., 53, 83-116.

Marusich W.L., Bauernfeind J.C., 1981. Oxycarotenoids in poultry feeds. In Bauernfeind J.C., (Ed.) Carotenoids as colorants and vitamin A precursors, 319-462. Acad. Press, New York.

Müller-Muló W., Rohrer G:, Medweth R., 1976. Schnellmethode zur quantitativen Bestimmung individueller Tocopherole in Ölen und Fetten. Fette. Seifen. Anstrichmittel., 78, 257-262.

Osadca M., Araujo M., De Ritter E., 1972. Determination of canthaxanthin in concentrates and feeds. J. Ass. Off. Analyt. Chem., 55, 110-113.

SCHIEDT K., LeUENBerger J.F., 1981. Retention, distribution and metabolism of astaxanthin in rainbow trout (Salmo gairdneri). 6th. Intern. Symposium on carotenoids, Liverpool, U.K. (Abstr.).

Snedecor G.W., Cochran W.G., 1967. Statistical methods. 6th ed. Iowa State Univ. Press. Ames (U.S.A.), 535 p.

Thommen H., Gloor U., 1965. Zum vorkommen von Ketocarotinoiden in der Forelle. Naturwiss., 52, 161-162.

VAldebouze P., LeVy B.R., 1972. Influence de l'agglomération et du stockage des aliments composés sur leur teneur en vitamine A. Ind. Alim. .4nim., 134, 71-77. 